Denis Gingras

Dominique Boivin

Diane Bilodeau

Julie Pelletier

Richard Béliveau

\title{
Les protéine carboxyle méthyltransférases des eucaryotes: deux classes distinctes d'enzymes
}

Les protéine carboxyle méthyltransférases sont des enzymes ubiquitaires qui catalysent le transfert d'un groupement méthyle de la S-adénosyl-L-méthionine à certains groupes carboxyles des protéines. Chez les eucaryotes, deux classes distinctes de carboxyle méthyltransférases ont été décrites. La première classe est représentée par les L-isoaspartate/D-aspartate méthyltransférases qui reconnaissent avec une forte affinité les résidus d'acide aspartique présents dans les protéines sous forme isomérisée ou racémisée et seraient impliquées dans la réparation de ces résidus. L'autre classe d'enzymes comporte les C-terminal carboxyl methyltransferases. Elles catalysent la méthylation du groupement carboxyl-terminal de protéines possédant une cystéine isoprénylée au niveau de leur portion C-terminale, tels plusieurs membres de la famille des petites protéines G. La méthylation participerait au recrutement des protéines $G$ cytosoliques isoprénylées par la membrane où elles deviennent actives. La méthylationdéméthylation pourrait donc servir de commutateur moléculaire pour régler le taux d'association des protéines $G$ isoprénylées à la membrane et, ainsi, l'activité des effecteurs de ces protéines, impliqués dans diverses fonctions biologiques telles que le transport vésiculaire, l'organisation du cytosquelette et la transduction des signaux hormonaux à travers la membrane.

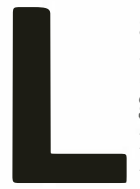

a modification covalente post-traductionnelle des groupes carboxyles par la méthylation représente une réaction extrêmement répandue dans le monde vivant. Dans cette réaction, les carboxyle méthyltransférases transfèrent le groupement méthyle de la S-
adénosyl-I-méthionine (AdoMet ou SAM) aux groupes carboxyles des protéines, avec formation d'un ester et neutralisation de la charge négative initialement portée par la protéine cible [1] (figure 1). L'ester formé est hydrolysé spontanément ou par l'action d'une méthylestérase, ce qui permet la production de 


\section{RÉFÉRENCES}

1. Barten DM, O'Dea RF. The function of protein carboxylmethyltransferase in eucaryotic cells. Life Sci 1990 ; 47 : 181-94.

2. Clarke S. Protein carboxyl methyltransferases: two distinct classes of enzymes. Ann Rev Biochem 1985; 54: 479-506.

3. O'Connor CM, Clarke S. Specific recognition of altered polypeptides by widely distributed methyltransferases. Biochem Biophys Res Comm 1985; 132: 1144-50.

4. Lowenson JD, Clarke S. Recognition of D-aspartyl residues in polypeptides by the erythrocyte L-isoaspartyl/D-aspartyl protein methyltransferase: implications for the repair hypothesis. J Biol Chem 1992; 267 : 5985-95.

5. Ingrosso D, Fowler AV, Bleibaum J, Clarke S. Sequence of the D-aspartyl/Lisoaspartyl protein methyltransferase from human erythrocytes. J Biol Chem 1989; 264 : 20131-9.

6. Henzel WJ, Stults JT, Hsu CA, Aswad DW. The primary structure of a protein carboxyl methyltransferase from bovine brain that selectively methylates Lisoaspartyl sites. J Biol Chem 1989; 264 : 15905-11.

7. Fu JC, Ding L, Clarke S. Purification, gene cloning, and sequence analysis of an L-isoaspartyl protein carboxyl methyltransferase from Escherichia coli. I Biol Chem 1991 ; 266 : 14562-72.

8. MacLaren DC, Kagan RM, Clarke S. Alternative splicing of the human isoaspartyl protein carboxyl methyltransferase RNA leads to the generation of a $\mathrm{C}$ terminal -RDEL sequence in isozyme II. Biochem Biophys Res Comm 1992; 185 : 277-83.

9. Potter SM, Johnson BA, Henschen A, Aswad DW, Guzzetta AW. The type II isoform of bovine brain protein L-isoaspartyl methyltransferase has an endoplasmic reticulum retention signal (...RDEL) at its Cterminus. Biochemistry $1992 ; 31$ : 6339-47.

10. Romanik EA, Ladino CA, Killoy LC, D'Ardenne SC, O'Connor CM. Genomic organization and tissue expression of the murine gene encoding the protein $\beta$ aspartate methyltransferase. Gene 1992 ; 118: 217-22.

11. Gingras D, Ménard P, Béliveau R. Protein carboxyl methylation in kidney brush border membranes. Biochim Biophys Acta $1991 ; 1066: 261-7$.

12. Gingras D, Boivin D, Béliveau R. Asymmetrical distribution of $\mathrm{L}$-isoaspartyl protein carboxyl methyltransferases in the plasma membranes of rat kidney cortex. Biochem J 1994; 297 : 145-50. méthanol et le retour de la protéine à sa structure originelle. La Sadénosyl-L-homocystéine (AdoHcy ou SAH), formée à la suite du transfert du groupement méthyle, agit comme inhibiteur compétitif des méthyltransférases. Chez les eucaryotes, les carboxyle méthyltransférases peuvent être divisées en deux grands types: les L-isoaspartate/Daspartate méthyltransférases et les Cterminal carboxyl methyltransferases, selon la nature du groupement carboxyle méthylé [1] (figure 2).

\section{Les carboxyle méthyltransférases de type II (L-isoaspartate) $D$-aspartate méthyltransférases)}

Les L-isoaspartate/D-aspartate méthyltransférases sont des enzymes largement distribuées dans le monde vivant, détectées jusqu'à présent dans quatre des cinq règnes vivants (Monères, Champignons, Plantes et Animaux) [1]. Les carboxyle méthyl- transférases II ont été purifiées du cytosol d'une variété de tissus animaux, végétaux et bactériens et, dans la plupart des cas, l'enzyme purifiée a un poids moléculaire variant entre 26 et $29 \mathrm{kDa}$, une constante d'affinité apparente $\left(\mathrm{K}_{\mathrm{m}}\right)$ pour le donneur de méthyle AdoMet de 1 à $3 \mu \mathrm{M}$ et une constante d'inhibition $\left(K_{i}\right)$ pour l'AdoHcy de 0,5 à $1,5 \mu \mathrm{M}$ [2]. Toutes ces enzymes ont une faible spécificité, reconnaissant une grande variété de peptides synthétiques et de protéines possédant des résidus $\mathrm{L}$-isoaspartates et $\mathrm{D}$ aspartates (ovalbumine, భglobulines, gélatine, certaines hormones hypophysaires) $[3,4]$. Le fractionnement des protéines cytosoliques d'une variété de tissus par chromatographie échangeuse d'ions ou par focalisation isoélectrique a mis en évidence la présence d'isoformes des carboxyle méthyltransférases II. Ainsi, dans le cytosol isolé d'érythrocytes humains et de cerveau de bœuf, deux formes de carboxyle méthyltransférases II ayant des points isoélectriques de 6.5

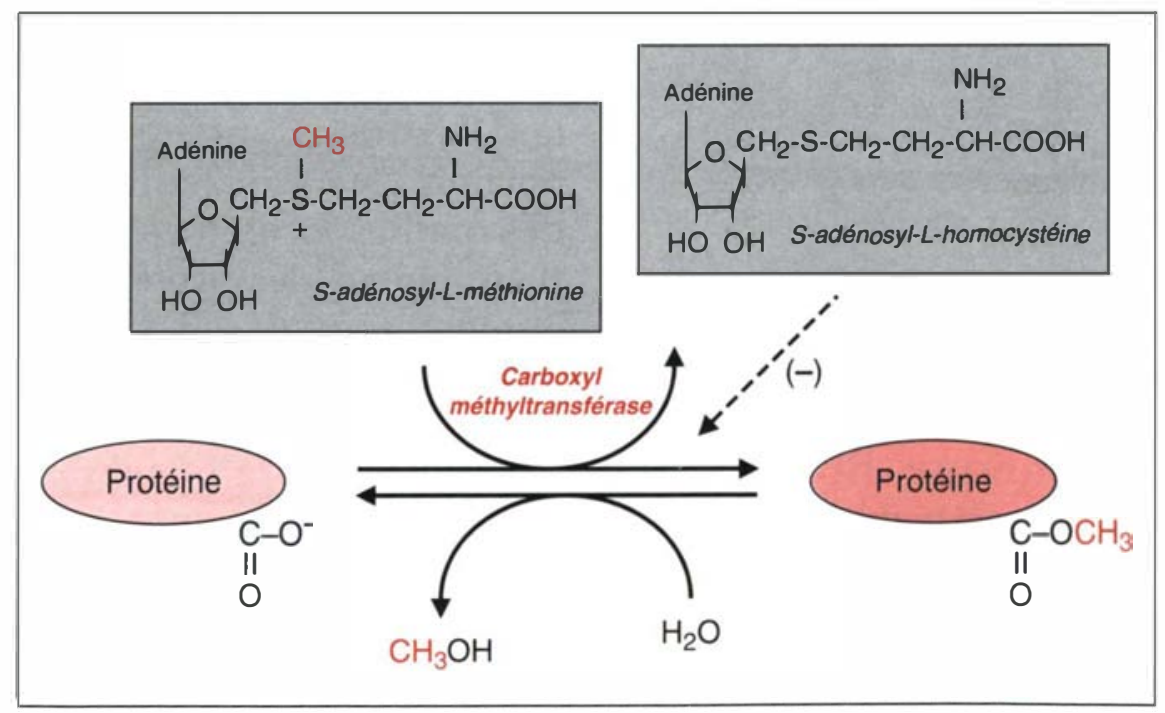

Figure 1. Modification covalente réversible des protéines par la méthylation des groupes carboxyles. Dans la réaction de méthylation, les protéine carboxyle méthyltransférases catalysent le transfert du groupement méthyle porté par le donneur S-adénosyl-L-méthionine (AdoMet ou SAM) à certains groupes carboxyles des protéines. La réaction de transfert permet la formation d'un ester de méthyle sur la protéine et la production de $S$ adénosyl-L-homocystéine (AdoHcy ou SAH), un inhibiteur compétitif des réactions de transméthylation. L'ester formé peut être hydrolysé de façon spontanée ou enzymatique, produisant dans les deux cas du méthanol. L'activité des carboxyle méthyltransférases est déterminée expérimentalement en utilisant de l'AdoMet tritiée et en mesurant la production de méthanol radioactif libéré par une hydrolyse alcaline des esters formés sur les protéines. 
(isoenzyme l) et de 5.5 (isoenzyme II) ont été identifiées, et les deux formes purifiées jusqu'à homogénéité $[5,6]$. La détermination de la séquence en acides aminés de ces carboxyle méthyltransférases II a révélé que l'isoenzyme I de la carboxyle méthyltransférase II d'érythrocytes est une protéine de 226 acides aminés d'un poids moléculaire de $24575 \mathrm{Da}$ [5], alors que la même isoenzyme isolée du cerveau de bœuf contient 226 acides aminés, pour un poids moléculaire de 24500 Da [6]. Les deux enzymes ont une très grande similitude, avec seulement 6 acides aminés divergents, et une similitude de $97 \%$. La carboxyle méthyltransférase II de $E$. coli est, quant à elle, une protéine de 207 résidus, d'un poids moléculaire de $23128 \mathrm{Da}$. La séquence dérivée de la protéine purifiée de $E$. coli montre une identité avec la carboxyle méthyltransférase II des eucaryotes dans 69 des 224 sites comparables, et une similitude de $31 \%$ [7]. Récemment, les séquences des isoenzymes II (formes plus aci- des) des carboxyle méthyltransférases II des érythrocytes et du cerveau ont été déterminées. Dans les deux cas, la seule différence notée entre les formes acide et basique se situe au niveau de la portion C-terminale de la protéine qui, pour l'isoenzyme I, se termine par Arg-Trp-Lys-COOH (-RWK) alors que celle de l'isoenzyme II se termine par ArgAsp-Glu-Leu-COOH (-RDEL) [8, 9]. La signification de la présence de ces isoformes de carboxyle méthyltransférases II dans plusieurs tissus n'est pas claire, mais l'obtention de clones codant pour l'enzyme pourrait permettre de mieux comprendre le phénomène. Un de ces clones, contenant la séquence codante entière de la carboxyle méthyltransférase II, a été isolé d'une banque génomique construite à partir de l'ADN de testicules de souris [10]. Le gène de l'enzyme comporte 7 exons, les exons 4,6 et 7 contenant des régions qui ont une homologie de séquence avec d'autres méthyltransférases et enzymes utilisant l'AdoMet. L'analyse du clone a révélé que les $\mathrm{ARNm}$ précurseurs subissent un épissage alternatif au niveau du dernier exon du gène. De plus, plusieurs ARNm sont détectés par Northern blot de l'ARN extrait du cerveau, des testicules, du foie et du rein [10]. Ces résultats suggèrent donc que les différentes isoformes des carboxyle méthyltransférases II observées dans plusieurs tissus proviennent d'un même gène. L'épissage alternatif au niveau du dernier exon est compatible avec les différences de structure entre les isoformes I et II des érythrocytes et du cerveau, différences localisées dans la portion C-terminale de ces protéines $[8,9]$.

C'est au niveau du cytosol que se situe la source majeure de l'activité carboxyle méthyltransférase II, et ce, dans tous les tissus étudiés jusqu'à présent. Selon certains auteurs, l'enzyme serait strictement cytosolique [2], et la grande majorité des études sur cette enzyme ont porté sur la caractérisation de cette forme soluble. Cependant, nous avons récemment identifié des formes de

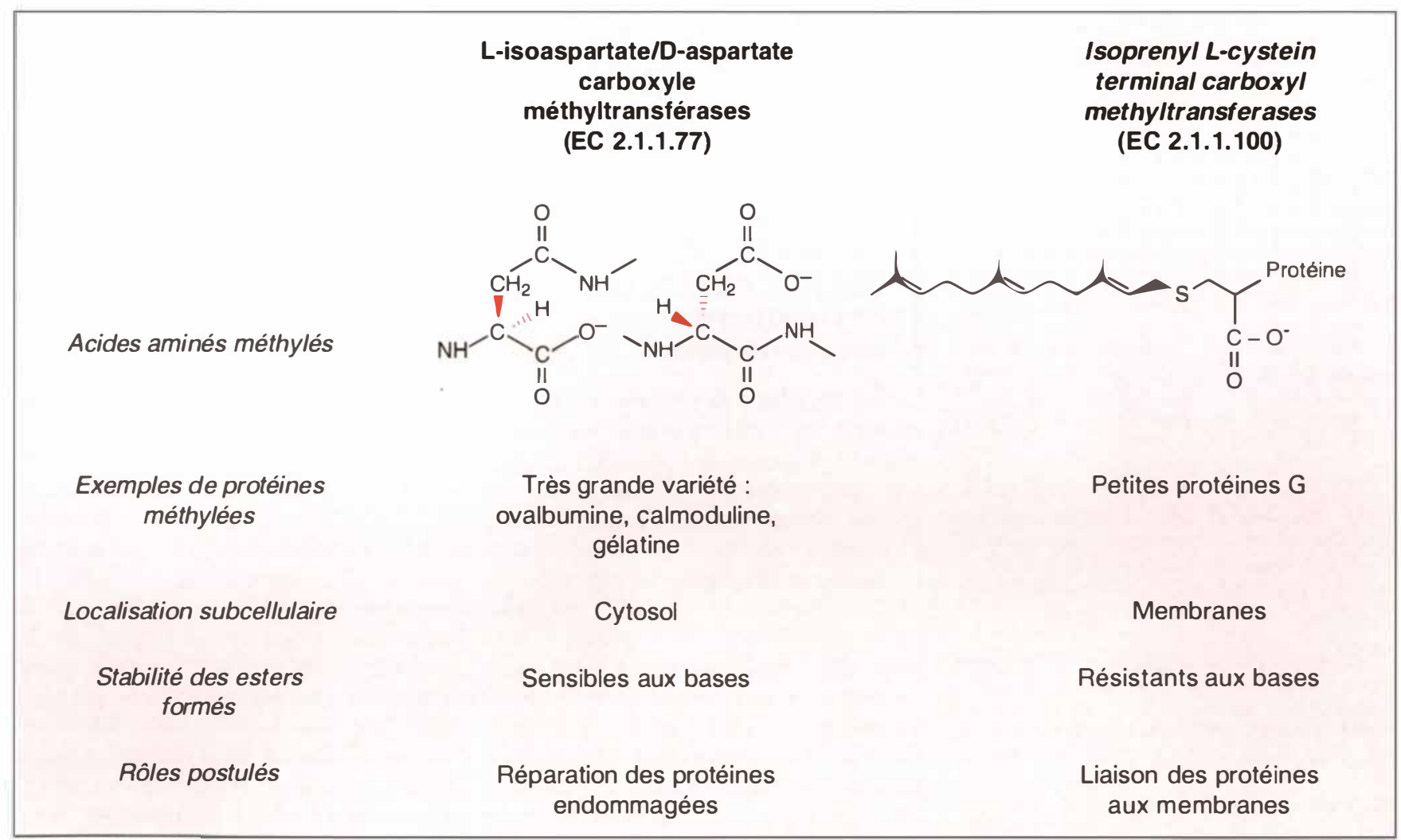

Figure 2. Les protéine carboxyle méthyltransférases des eucaryotes. 


\section{RÉFÉRENCES}

13. Boivin D, (ingras D, Béliveau R. Purification and characterization of a membrane-bound protein carboxyl methyltransferase from rat kidney cortex. I Biol Chem 1993; 268: 261()-5.

14. Clarke S, O'Connor C.M. Do eucaryotic carboxyl methyltransferases regulate protein function? Trends Biochem Sci 1983; 8 : 391-4.

15. McFadden PN, Clarke S. Methylation at D-aspartyl residues in erythrocytes : possible step in the repair of aged membrane proteins. Proc Natl Acad Siri USA 1982; 79 246()-4.

16. Aswad DW. Stoichiomeuric methylation of porcine adrenocorticotropin by protein carboxyl methyltransferase requires deamidation of asparagine 25: evidence for methylation at the $\alpha$-carboxyl group of atypical I-isoaspartyl residues. If Biol Chem 1984 ; 259 : $10714-21$.

17. Murray ED, Clarke S. Synthetic peptide substrates for the erythrocyte protein carboxyl methyltransferase: detection of a new site of methylation at isomerized Laspartyl residues. I Biol Chem 1984; 259 : $10722-32$.

18. Lowenson JD, Clarke S. Spontaneous degradation and enzymatic repair of aspartyl and asparaginyl residues in aging red cell proteins analyzed by computer simulation. Gerontol 1991; 37 : 128-51.

19. (jeiger T, Clarke S. Deamidation, isomerization, and racemization at asparaginyl and aspartyl residues in peptides succinimide-linked reactions that contribute to protein degradation. I Biol Chem 1987; 262 : 785-94.

20. Johnson BA, Langmark EL, Aswad DW. Partial repair of deamidation-damaged calmodulin by protein carboxyl methyltransferase. I Biol Chem 1987; 262: 12283-7.

21. McFadden PN, Clarke S. Conversion of isoaspartyl peptides to normal peptides: implications for the cellular repair of damaged proteins. Proc Nall Acad Sci USA 1987 ; 84 : 2595-9.

22. Johnson BA, Murray ED, Clarke S, Glass DB, Aswad DW. Protein carboxyl methyltransferase facilitates conversion of atypical L-isoaspartyl peptides to normal Laspartyl peptides. J Biol Chem 1987; 262 : 5622-9.

23. Li C, Clarke S. A protein methyltransferase specific for altered aspartyl residues is important in Escherichia coli stationaryphase survival and heat-shock resistance. Proc Nall Acad Sici USA 1992; 89 : 9885-9.

24. Ladino CA, O'Connor CM. Methylation of atypical protein aspartyl residues during the stress response of Hela cells. carboxyle méthyltransférases II liées aux membranes à bordure en brosse [11] et basolatérales [12] des cellules du cortex rénal, et nous avons réussi à purifier l'enzyme de la membrane à bordure en brosse 13]. Les enzymes membranaires sont fortement associées à la membrane puisque des concentrations élevées de détergents sont nécessaires à leur solubilisation [ 12 , 13]. Les différences entre les carboxyle méthyltransférases II membranaires et cytosoliques restent à caractériser car, une fois solubilisée, l'enzyme membranaire semble posséder les mêmes propriétés catalytiques et moléculaires que l'enzyme soluble et semble très homologue au niveau structural [13]. L'association à la membrane pourrait donc être due à une modification posttraductionnelle d'une enzyme précurseur soluble, ou pourrait être reliée à la présence de motifs structuraux au sein même de sa structure signalant son association à la membrane. Dans cette optique, il est intéressant de noter qu'une des carboxyle méthyltransférases II du cerveau et des érythrocytes (l'isoenzyme II) possède un motif (-RDEL) au niveau de sa portion C-terminale, motif retrouvé chez plusieurs protéines destinées à résider au niveau du réticulum endoplasmique $([8,9]$, $\mathrm{m} / \mathrm{s} n^{\circ} 11$, vol. 9, p. 1249).

\section{Implication de la méthylation dans le métabolisme des protéines endommagées}

La méthylation des protéines catalysée par les carboxyle méthyltransférases II présente des caractéristiques très particulières. I'une part, les esters formés sont très sensibles à l'hydrolyse alcaline et, d'autre part, le taux de méthylation est substoechiométrique, la grande majorité des substrats identifiés jusqu'à présent étant méthylés avec des stochiométries largement inférieures à 1,0 mole méthyle/mole polypeptide [2]. Ces propriétés sont difficilement compatibles avec un modèle de régulation des fonctions enzymatiques par méthylation [14]. Au niveau de plusieurs protéines on a identifié comme sites majeurs de méthylation des résidus d'acide aspartique possédant la conformation I) [15] et du L-isoaspartate, une autre forme de résidu aspartate altéré $[16,17]$, ce qui a ouvert un tout autre champ d'études quant au rôle potentiel de cette classe d'enzyme.

\section{Origine des résidus D-aspartates et L-isoaspartates}

L'asparagine et l'aspartate sont les résidus d'acides aminés les plus labiles retrouvés au sein des protéines, non seulement à cause de la réactivité de la chaîne amide ou carboxyle latérale, mais également parce que cette chaîne latérale possède une longueur susceptible de subir une attaque intramoléculaire par l'azote de la liaison peptidique adjacente [18]. Cette réaction entraîne la déamidation de l'asparagine ou la déshydratation de l'aspartate, produisant dans les deux cas un intermédiaire succinimide cyclique [19] (figure 3). Le succinimide est instable et son hydrolyse spontanée produit un résidu aspartate normal ou un produit de transpeptidation isoaspartate dans lequel le lien peptidique est formé par le groupement carboxyle $\beta$ de la chaîne latérale. Ce processus non enzymatique est également accompagné d'une racémisation importante de l'intermédiaire succinimide, engendrant les résidus D-aspartate et Disoaspartate. Les carboxyle méthyltransférases II de différentes sources sont très réactives envers les résidus L-isoaspartates présents dans divers peptides et protéines [3]. Par exemple, la conversion chimique d'un hexapeptide non reconnu par l'enzyme en un isopeptide transforme celui-ci en un excellent substrat, méthylé avec une stœechiométrie approchant 1,0 [17]. La méthylation du ID-aspartate et du Lisoaspartate semble catalysée par la même enzyme, mais l'affinité de la carboxyle méthyltransférase II pour ces deux résidus est cependant très distincte. Ainsi, alors que les constantes d'affinité de l'enzyme envers divers peptides synthétiques contenant un résidu L-isoaspartate sont de l'ordre de $10^{-6} \mathrm{M}$, les plus fortes constantes d'affinité pour des pepti- 


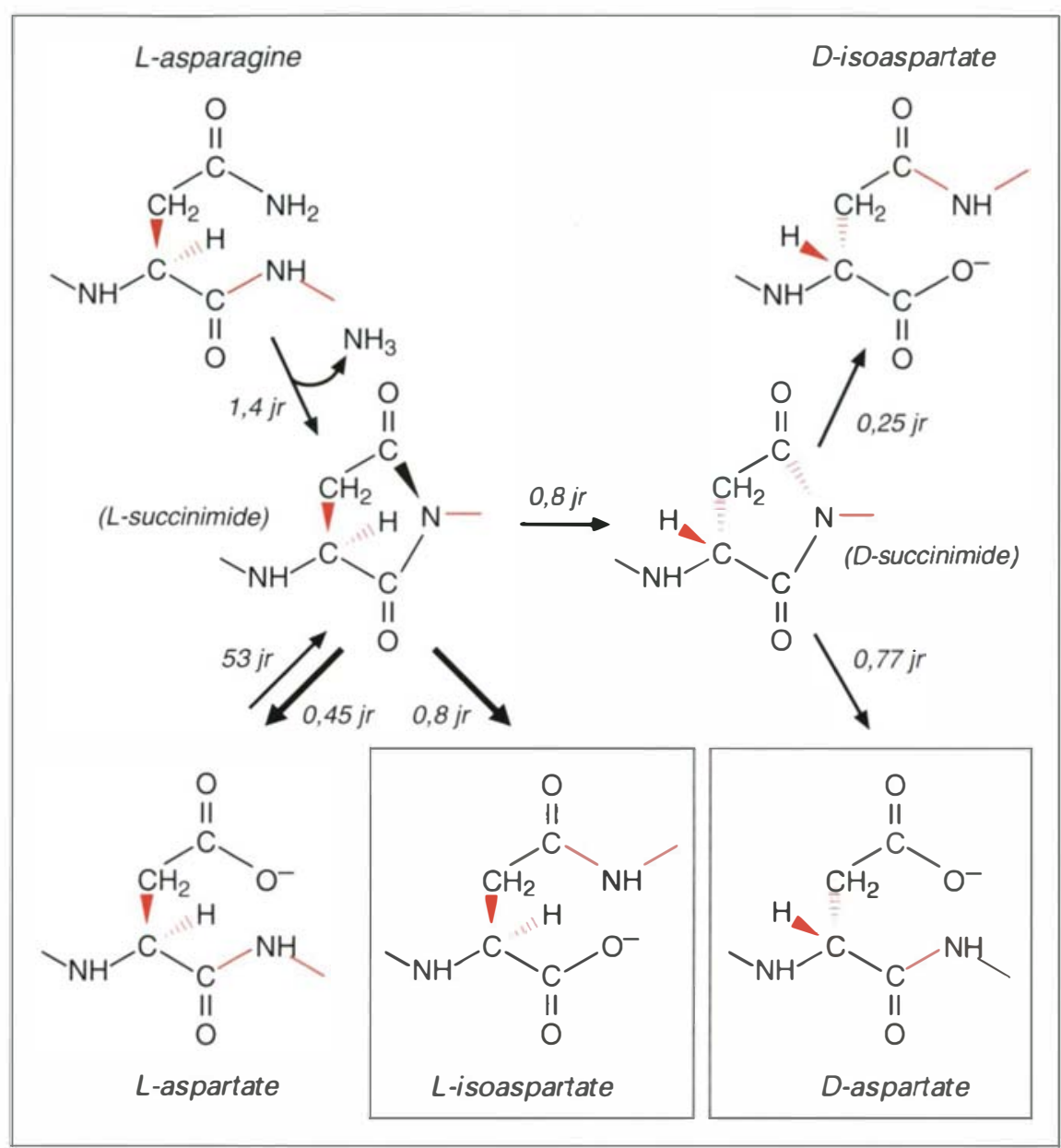

Figure 3. Formation spontanée des résidus L-isoaspartate et Daspartate à partir des résidus asparagine et aspartate normaux. En conditions physiologiques normales, les résidus asparagine et aspartate sont susceptibles de subir une attaque intramoléculaire de l'azote de la liaison peptidique, avec formation d'un intermédiaire cyclique à cinq membres, le succinimide. Cet intermédiaire est très instable et s'hydrolyse de façon spontanée pour former un résidu aspartate normal ou encore un produit de transpeptidation, L-isoaspartate, dans lequel la liaison peptidique est maintenant formée par le groupement carboxyle de la chaîne latérale. De plus, l'intermédiaire succinimide est sujet à la racémisation, provoquant la formation de D-isoaspartate et de D-aspartate. La formation de ces résidus altérés est fortement influencée par la structure tridimensionnelle de la protéine ainsi que par la séquence en acides aminés adjacents à l'asparagine et à l'aspartate. Les études réalisées jusqu'à présent suggèrent que seuls les résidus L-isoaspartate et D-aspartate (encadrés) sont reconnus par la carboxyle méthyltransférase II.

des contenant un résidu $\mathrm{D}$-aspartate sont de l'ordre de $10^{-3} \mathrm{M}(2,5$ à $\left.4,8 \cdot 10^{-3} \mathrm{M}\right)$, soit une affinité de l'enzyme de 700 à 10000 fois plus faible [4].

Rôle de la méthylation dans la réparation des protéines endommagées

La présence de résidus L-isoaspartate et D-aspartate semble avoir des effets $\mathrm{m} / \mathrm{s} n^{\circ} 1$ vol. 10 , janvier 94 résidus pourrait promouvoir leur réparation et diminuer ainsi les dommages causés à la structure et à la fonction des protéines par le vieillissement [15] (figure 4). Dans ce modèle, la méthylation par la carboxyle méthyltransférase II du résidu L-isoaspartate provoque l'activation du carboxyle et la formation spontanée d'un intermédiaire succinimide. Celle-ci entraîne une déméthylation très rapide, compatible avec les données acquises in vivo et in vitro sur la stabilité des esters de méthyle formés sur les protéines. L'hydrolyse non enzymatique du succinimide produit du L-aspartate $(20 \%)$ et du L-isoaspartate $(80 \%)$, le carbone adjacent au carbone en position $\alpha$ étant plus susceptible à l'hydrolyse. I.e L-isoaspartate est disponible alors pour subir un autre cycle de réactions par sa méthylation, avec le résultat net d'une conversion de l'isopeptide en peptide normal [21]. Cie processus n'est cependant pas totalement efficace, limité qu'il est par la racémisation du carbone en position $\alpha$ du succinimide en I)-isoaspartate, non reconnu par l'enzyme [19, 21]. L'efficacité de cette voie de réparation, qui consiste en une réaction enzymatique et deux étapes non enzymatiques, a été montrée in vitro en utilisant des peptides synthétiques. Ainsi, l'incubation de trois peptides différents contenant un résidu L-isoaspartate avec la carboxyle méthyltransférase II purifiée et l'AdoMet à pH $7.4,37^{\circ} \mathrm{C}$, pour 48 heures, permet la conversion de $80 \%$ des résidus L-isoaspartates en résidus L-aspartates normaux [22].

Ces réparations pourraient avoir une signification physiologique importante; par exemple, la méthylation in vitro de la calmoduline permet une récupération substantielle de sa capacité à activer la kinase dépendante du calcium et de la calmoduline [20]. L'importance du mécanisme de réparation par la méthylation est soulignée par les résultats récents obtenus sur la carboxyle méthyltransférase II de E. coli. En effet, des souches de $E$. coli mutantes pour le gène $p c m$ qui code pour la carboxyle méthyltransférase II ne possédent donc pas d'activité de méthylation et survivent très mal 


\section{RÉFÉRENCES}

25. Clarke S. Protein isoprenylation and methylation at carboxyl-terminal cysteine residues. Annu Re Biochem 1992; 61: 355-86.

26. Clarke S, Vogel JP, Ieschenes RJ, Stock JB. Post-translational modification of the Ha-ras oncogene protein : evidence for a third class of protein carboxyl methyltransferases. Proc Natl Acad Sri ISA 1988 8.5 : $4643-7$.

27. Hrycyna CA, Sapperstein SK. Clarke S, Michaelis $\mathrm{S}$. The Saccharomyces cerevisiae STE 14 gene encodes a methyltransferase that mediates C-terminal methylation of afactor and Ras proteins. $E M B O / 1991$; 10 : 1699-709.

28. Stephenson RC, Clarke S. Characterization of a rat liver protein carboxyl methyltransferase involved in the maturation of proteins with the C.XXX C-terminal sequence motif. J Biol Chem 1992; 267 : 13314-9.

29. Boivin I), Potier M, Béliveau R. Functional size of C-terminal protein carboxyl methyltransferase from kidney basolateral plasma membrane. Biochim Biophys Acla 1994 (sous presse)

30. Volker C, Miller RA, Mccileary WR, et al. Eflects of farnesylcysteine analogs on protein carboxyl methylation and signal transduction. I Biol (.hem 1991; 266: 21515-22.

31. Volker C, I ane P, Kwee C, Johnson M, Stock JB. A single activity carboxyl methylates both farnesyl and geranylgeranyl cysteine residues. FEBSS I ell 1991; 295: 189-94.

32. Gingras (), Boivin I), Béliveau R. Subcellular distribution and guanine nucleotide dependency of COOH-terminal methylation in kidney cortex. Am J Physiol 1993 $265: 316-22$

33. Hancock JF, Cadwallader K, Marshall C.J. Methylation and proteolysis are essential for efficient membrane binding of prenylated $\mathrm{p} 21^{\mathrm{k}-\operatorname{ras}(\mathrm{B})} . E M B O / 1991 ; 10$ : $641-6$

34. Béranger F, Goud B, Tavitian A, Gunzburg J. Association of the Ras-antagonistic Rapl/Krev-l proteins with the Golgi complex. Proc Natl Acad Siri USA 1991; 88: $1606-10$.

35. Chavrier P, Parton RG, Hauri HP Simons K. Zerial M. Localization of low molecular weight GTP binding proteins to exocytic and endocytic compartunents. Cell $1990 ; 62: 317-29$

36. Chavrier P, Gorvel JP, Stelzer E, Simons K, Gruengerg .J, Zerial M. Hypervariable C-terminal domain of Rab proteins acts as a targeting sigmal. Nature $1991 ; 353$ :

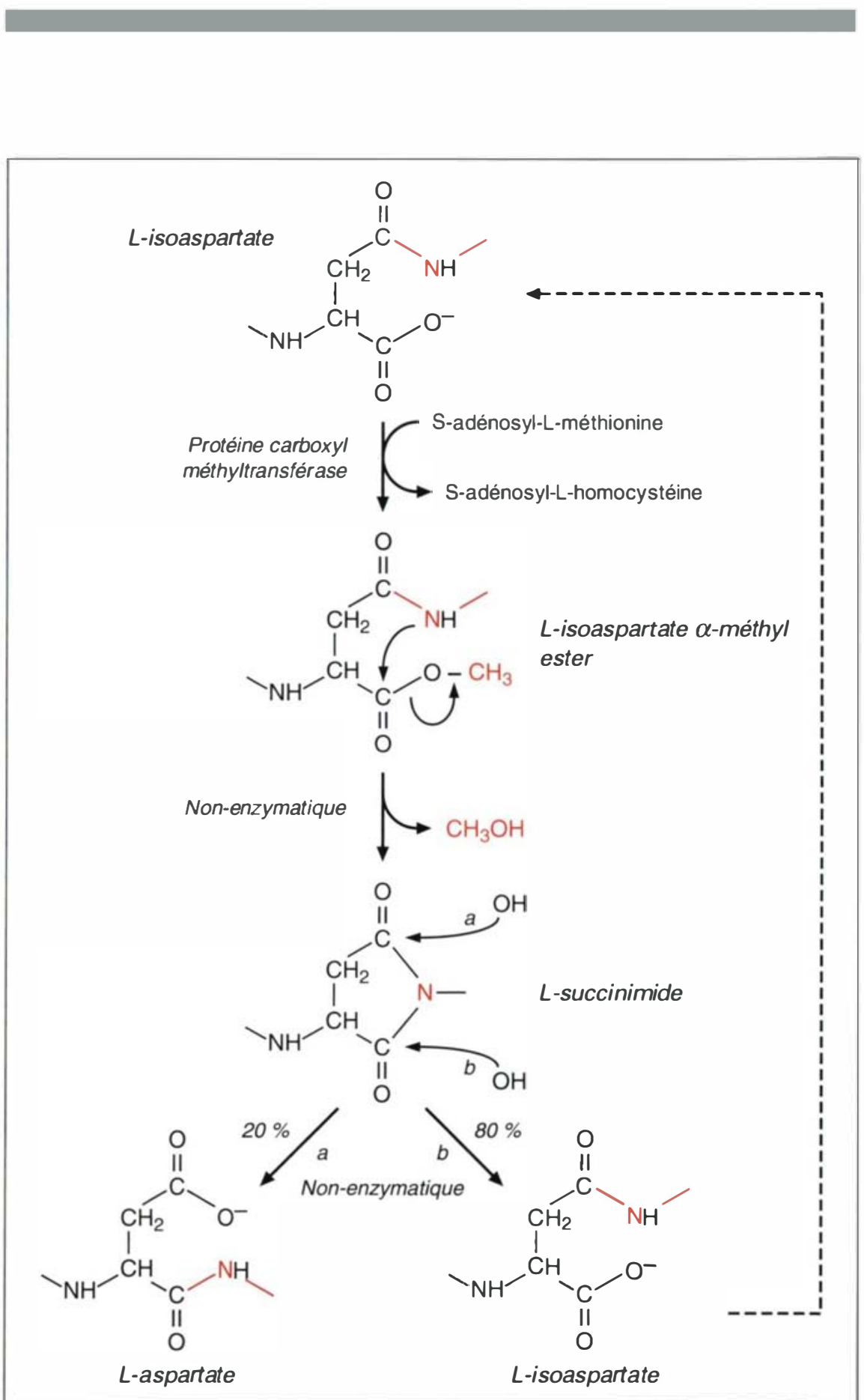

Figure 4. Modèle proposé pour la réparation des résidus altérés Lisoaspartates en résidus aspartates normaux. La réparation de résidus L-isoaspartates en aspartates normaux consiste en une réaction enzymatique et en deux réactions non enzymatiques. Dans un premier temps, le résidu L-isoaspartate est méthylé par la carboxyle méthyltransférase. Cette méthylation entraîne l'activation du groupement carboxyle en position $\alpha$ et favorise la formation d'un intermédiaire succinimide et la déméthylation très rapide de la protéine. L'intermédiaire succinimide est instable et son hydrolyse engendre un résidu aspartate "réparé" ainsi qu'un résidu Lisoaspartate "non réparé". Ce dernier peut alors subir un autre cycle de réactions, réparant encore en une fraction de résidus. La répétition de ce cycle permet donc une réparation substantielle de la protéine. 
lorsqu'elles sont exposées à des conditions favorisant l'accumulation de résidus altérés, tels le choc thermique ou la culture en phase stationnaire [23]. Chez les eucaryotes, l'incubation prolongée de cellules HeLa à une température de $42^{\circ} \mathrm{C}$ provoque une élévation importante $(80 \%)$ de l'activité de carboxyle méthylation [24], indépendante de la synthèse d'enzyme, probablement liée à une augmentation de résidus altérés qui peuvent être méthylés par la carboxyle méthyltransférase II [24]. Ces résultats suggèrent donc que la méthylation de résidus altérés présents sur les protéines est efficace in vivo, et pourrait contribuer au maintien de la fonction de ces dernières et réduire ainsi les dommages causés à la cellule par son vieillissement ou son exposition à des stress physiques.

\section{Les C-terminal carboxyl methyltransferases}

Les C-terminal carboxyl methyltransferases font partie d'un groupe d'enzymes impliquées dans la modification post-traductionnelle de protéines possédant une cystéine localisée dans une séquence $\mathrm{C}$-terminale terminée par CXXX, XCXC ou $X X C C$ (où $C$ représente une cystéine et $X$ n'importe quel acide aminé) [25]. L'isoprénylation et la méthylation des cystéines localisées dans la séquence consensus CXXX ont été particulièrement étudiées et le schéma réactionnel postulé est illustré dans la figure 5. Dans un premier temps, le précurseur cytosolique immature de la protéine possédant le motif CXXX est isoprénylé sur la cystéine C-terminale par l'addition d'un groupement farnésyle (isoprène à 15 carbones) ou géranylgéranyle (isoprène à 20 carbones). Puis ce dérivé isoprénylé subit le clivage protéolytique du tripeptide C-terminal, ce qui a pour effet d'exposer le groupement carboxyle de la cystéine isoprénylée, maintenant en position $\mathrm{C}$-terminale. Ce groupement carboxyle est méthylé par la suite sous l'action d'une carboxyle méthyltransférase qui transfère le groupement méthyle de l'AdoMet au groupement carboxyle. Le résultat net de ces réac-

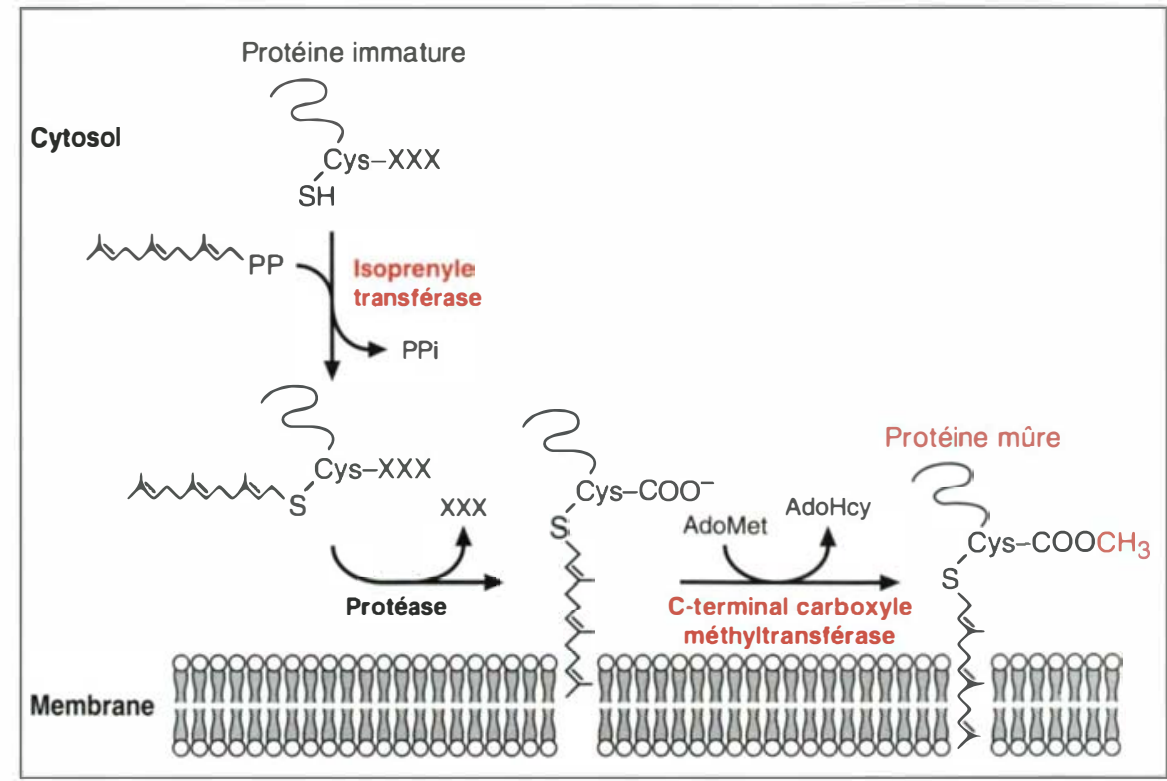

Figure 5. Modifications post-traductionnelles des protéines à motif CXXX. Les protéines possédant le motif CXXX loù $C=$ cystéine, $\mathrm{X}=$ n'importe quel acide aminé) sont synthétisées sous forme de précurseurs cytosoliques immatures. Ces précurseurs sont dans un premier temps isoprénylés par des groupements isoprènes farnésyles ou géranylgéranyles (farnésyle représenté sur la figure), ce qui permet une association faible à la membrane. La protéine isoprénylée subit alors le clivage protéolytique du tripeptide C-terminal par une endoprotéase membranaire, ce qui provoque l'apparition d'un groupement carboxyle libre au niveau de la cystéine isoprénylée. Ce groupement carboxyle est par la suite méthylé par l'action de la C-terminal carboxyl methyltransferase, également membranaire, renforçant ainsi l'association de la protéine à la membrane. AdoMet: S-adénosyl-Lméthionine; AdoHcy: S-adénosyl-L-homocystéine.

tions est la formation d'un Sisoprényl cystéine $\alpha$-méthyl ester en position C-terminale de la protéine [25].

Les C-terminal carboxyl methyltransferases ont été initialement mises en évidence lors de l'étude de la structure de peptides d'accouplement de Tremella brasiliensis, une souche de champignons basidiomycètes, et du facteur a de $S$. cerevisiae. En effet, ces peptides possèdent en position $\mathrm{C}$ terminale une cystéine qui est modifiée par l'addition d'un résidu farnésyle et d'un ester de méthyle. La similitude de séquence de la portion C-terminale de ces peptides et de celles retrouvées au sein des protéines Ras suggérait une possible méthylation de ces dernières, ce qui fut ensuite démontré [26]. La stabilité du lien ester en condition modérément alcaline suggérait l'action d'une classe de méthyltransférase distincte des L-isoaspartate méthyltransférases, reconnaissant le groupement C-terminal carboxyl [26]. La nature de cette enzyme demeure encore largement inconnue. Chez $S$. cerevisiae, la méthylation du facteur a et des protéines Ras dépend de l'expression du gène STE 14, gène codant pour une protéine de 239 acides aminés $(\sim 28 \mathrm{kDa})$ avec plusieurs segments transmembranaires potentiels [27]. Chez les mammifères, l'enzyme n'a pu être purifiée jusqu'à présent étant donné sa très grande sensibilité aux détergents [28]. Par la méthode d'inactivation par les radiations ionisantes nous avons étudié la taille de la C-terminal carboxyl methyltransferase des membranes basolatérales du rein et montré que cette enzyme possède une taille fonctionnelle de $98 \mathrm{kDa}$, ce qui fait envisager une nature multimérique [29].

L'utilisation de substrats synthétiques farnésylés a permis d'étudier plus en détail les C-terminal carboxyl 


\section{RÉFÉRENCES}

37. Khosravi-Far R, (iox AI), Kitto $K$, I)er C.J. Protein prenylation: key to Ras func tion and cancer intervention? Cell (irowth I)iff $1992 ; 3$ : 461-9.

38. Kohl NE, Mosser SI), De Solms J, el al. Selective inhibition of Ras-dependent uans formation by a farnesyltransferase inhibitor. Science 1993; 260) 1934-7.

39. James GL, Coldstein JI, Brown MS, et al. Benzodiazepine peptidomimetics : potent inhibitors of Ras farnesylation in animal cells. Science 1993; 260: 1937-42.

40. Takai Y, Kaibuchi K, Kikuchi A, Kawata M. Small (;TP-binding proteins. Int Ret Cytol $1992 ; 133$ : 187-230.

41. Kahn A. I a transmission du signal en amont et en aval de Ras. médecine/sciences $1992 ; 8: 1097-9$.

42. Mizuno $\mathrm{T}$, Kaibuchi $\mathrm{K}$, Yamamoto $\mathrm{T}$, et al. A stimulatory (;I)P/(;TP exchange protein for smg p21 is active on the posttranslationnally processed form of c-Ki-Ras p21 and RhoA p21. Proc Nall Acad Sci USA 1991 ; 88 : 6442-6.

43. Hori Y, Kikuchi A, Isomura $M$, et al. Post-translational modifications of the C. terminal region of the Rho protein are important for its interaction with membranes and the stimulatory and inhibitory (;DP/C;TP exchange proteins. Oncogene $1991 ; 6: 515-22$.

44. Marshall (J. Protein prenylation: a mediator of protein-protein interactions. Science 1993 ; 259: 1865-6.

45. Backlund PS, Aksamit RR. (;uanine nucleotide-dependent carboxyl methylation of mammalian membrane proteins. I Biol Chem 1988; 263: 15864-7.

46. Huzoor-Akbar, Winegar DA, Iapetina E(;. Carboxyl methylation of platelet Rap l proteins is stimulated by guanosine 5 - $-(3 \mathrm{C}$ thio) triphosphate. J Biol C.hem 1991; 266: 4.387-91.

47. Huzoor-Akbar, Wang W, Kornhauser R, Volker (;, Stock JB. Protein prenylcysteine analog inhibits agonist-receptormediated signal transduction in human platelets. Proc Nall Acad Sci USA 1993; 90: 868-72.

48. Philips MR, Pillinger MH, Staud R, et al. ('arboxyl methylation of Ras-related proteins during signal transduction in neutro- methyltransferases. Par exemple, le peptide Sfarnésyl-LARYKC*, dérivé de la séquence de la protéine Ras de Drosophila melanogaster, est un excellent substrat pour l'enzyme [28]. L'enzyme reconnaît également des versions extrêmement simplifiées de substrats isoprénylés, telle la $\mathrm{N}$ acétyl-farnésyl-cystéine (AFC) [30]. L.es études réalisées jusqu’à présent suggèrent que la portion isoprénylée est le principal déterminant de reconnaissance de l'enzyme, la suructure même de la protéine étant beaucoup moins importante. Cependant, la C-terminal carboxyl methyltransferase ne semble pas distinguer la nature de ce groupement isoprène, reconnaissant aussi bien le farnésyl que le géranylgéranyl [31]. L'étude de la méthylation de ces différents substrats synthétiques a révélé que l'activité de méthylation était associée à la fraction membranaire de $S$. cerevisiae et de différents tissus de mammifères [28, 30, 32]. Au niveau du tissu hépatique, l'activité de méthylation co-migre avec des marqueurs enzymatiques du réticulum endoplasmique (RE) sur gradient de Percoll, et non avec ceux de la membrane plasmique, suggérant une localisation préférentielle dans le RE [28]. Nous avons cependant montré que ces enzymes étaient présentes dans plusieurs compartiments membranaires au niveau du cortex rénal, ce qui pourrait permettre la méthylation de protéines isoprénylées localisées dans différentes membranes cellulaires [32]. Cela est en accord avec un modèle de régulation des fonctions enzymatiques par méthylationdéméthylation, la protéine déméthylée pouvant être reméthylée in situ [30]. En effet, des différentes étapes de maturation des protéines à motif CXXX, la méthylation est potentiellement la seule réaction réversible et pourrait donc être soumise à une régulation [30].

\section{Rôles de l'isoprénylation/ méthylation}

Comme la majorité des protéines isoprénylées et méthylées identifiées jusqu’à présent sont associées aux membranes, il a été proposé que ces

* I.: leu; $A$ : ala $; R:$ arg; $Y:$ tyr; K: lys ; C: cys. modifications soient impliquées dans cette association [25]. En effet, les protéines normalement associées aux membranes se retrouvent dans le cytosol lorsque les cellules sont traitées par la lovastatine, un inhibiteur de l'isoprénylation. De plus, des protéines Ras, farnésylées mais non méthylées, voient leur association à la membrane réduite de moitié [33], ce qui suggère qu'en plus de l'isoprénylation, la méthylation est importante pour l'association aux membranes. Ia neutralisation de la charge négative portée par le groupement carboxyle de la cystéine pourrait renforcer la liaison hydrophobe de l'isoprène avec la bicouche lipidique. L'addition d'un groupement méthyle pourrait, de plus, permettre la reconnaissance d'une protéine réceptrice et favoriser une interaction spécifique protéineprotéine [30]. Par exemple, des souches de $S$. cerevisiae mutantes du gène STE14 (codant pour une méthyltransférase inactive) possèdent un facteur a de couplage biologiquement inactif qui ne peut interagir avec son récepteur [27]. Ces résultats suggèrent donc que le groupement méthyle pourrait agir comme déterminant de la reconnaissance d'un récepteur spécifique des protéines isoprénylées. L'existence de tels récepteurs est également suggérée par la localisation des petites protéines $G$ au niveau des différents organites cellulaires. Par exemple, les protéines Rapl et Rab5, possédant toutes deux un groupement géranylgéranyle, sont respectivement retrouvées au niveau de l'appareil de Golgi [34] et des endosomes précoces [35]. Il est difficile d'imaginer de quelle façon une telle distribution subcellulaire pourrait exister si le seul déterminant d'association de ces protéines avec les membranes était l'acquisition d'un degré d'hydrophobicité. Dans le cas des protéines Rab, le motif C-terminal est essentiel à l'association de ces protéines aux membranes, mais est insuffisant pour spécifier le ciblage des protéines au compartiment cellulaire où elles exercent leurs fonctions. Ce ciblage spécifique serait dû au domaine hypervariable adjacent aux cystéines C-terminales et pourrait donc faire intervenir des récep- 
teurs spécifiques [36]. L'identification de tels récepteurs pourrait permettre, d'une part, une meilleure compréhension du rôle de l'isoprénylation et de la méthylation dans l'attachement des protéines aux membranes et, d'autre part, de permettre le design d'antagonistes de ces récepteurs pouvant bloquer spécifiquement l'association à la membrane de protéines ayant un potentiel oncogénique et éliminer ainsi leur potentiel transformant. Cette perte de pouvoir transformant après inhibition de l'isoprénylation par des manipulations génétiques et pharmacologiques a été mise en évidence pour le produit d'oncogène ras ([37-39], m/s no 8-9, vol. 9, p. 996).

Un rôle de l'isoprénylation et de la méthylation dans les interactions protéine-protéine ést également suggéré par l'étude des protéines qui règlent l'échange des nucléotides sur les petites protéines $G$. Les petites protéines $G$ ont pour caractéristique d'osciller entre un état actif, lié au GTP et un état inactif, lié au GIP. Lorsqu'elles sont liées au (II)P, ces protéines interagissent avec des facteurs d'échange inhibiteurs (GI)I) ou stimulateurs (GDS) de la dissociation du GDP, alors que dans leurs états liés au GTP elles interagissent avec des protéines stimulant l'hydrolyse du GTP (GAP) [40,41] (figure 6). Certaines de ces interactions semblent requérir la présence de groupements isoprènes et méthyles. Ainsi, la modification post-traductionnelle par méthylation et isoprénylation des petites protéines $\mathrm{G}$ Ki-Ras ${ }^{4 \mathrm{~B}}$, RhoA et Rap semble essentielle à leur interaction avec une protéine GDS reconnaissant ces différentes protéines [42]. I'importance de ces modifications a également été observée pour l'interaction des petites protéines $G$ Rab3A et Rho/Rac avec leurs protéines GDI [43]. Cela est d'autant plus intéressant que les protéines GII ont la propriété de provoquer la dissociation des petites protéines $G$ des membranes vers le cytosol, probablement en enfouissant les groupements isoprène et méthyle dans une pochette hydrophobe de leur structure. Ces protéines pourraient donc s'avérer être des régulateurs du cycle d'associationentre les membranes et le cytosol, cycle qui serait dépendant de la modification post-traductionnelle des petites protéines $G$ par isoprénylation et méthylation [44] (figure 6). Que la méthylation des petites protéines $G$ joue un rôle dans la régulation de certaines fonctions enzymatiques est suggéré par la démonstration que la méthylation de ces protéines est fortement stimulée par la présence d'analogues non hydrolysables du GTP [32, 45, 46] ; l'enzyme reconnaîtrait préférentiellement l'état activé, susceptible d'interagir avec les effecteurs de ces protéines. dissociation des petites protéines

De plus, l'AFC, un substrat synthétique reconnu par les C-terminal carboxyl methyltransferases, provoque une inhibition importante du chimiotactisme des macrophages localisés dans le péritoine de souris [30], ainsi que de la réponse des plaquettes à divers agonistes [47]. L'addition d'AFC' à des neutrophiles humains provoque une baisse importante de la production d'anion superoxide induite par le facteur chimiotactique formyl-méthionylleucyl-phénylalanine [48]. Or, la production de superoxide par la NADPH oxidase de ces cellules nécessite la participation de petites

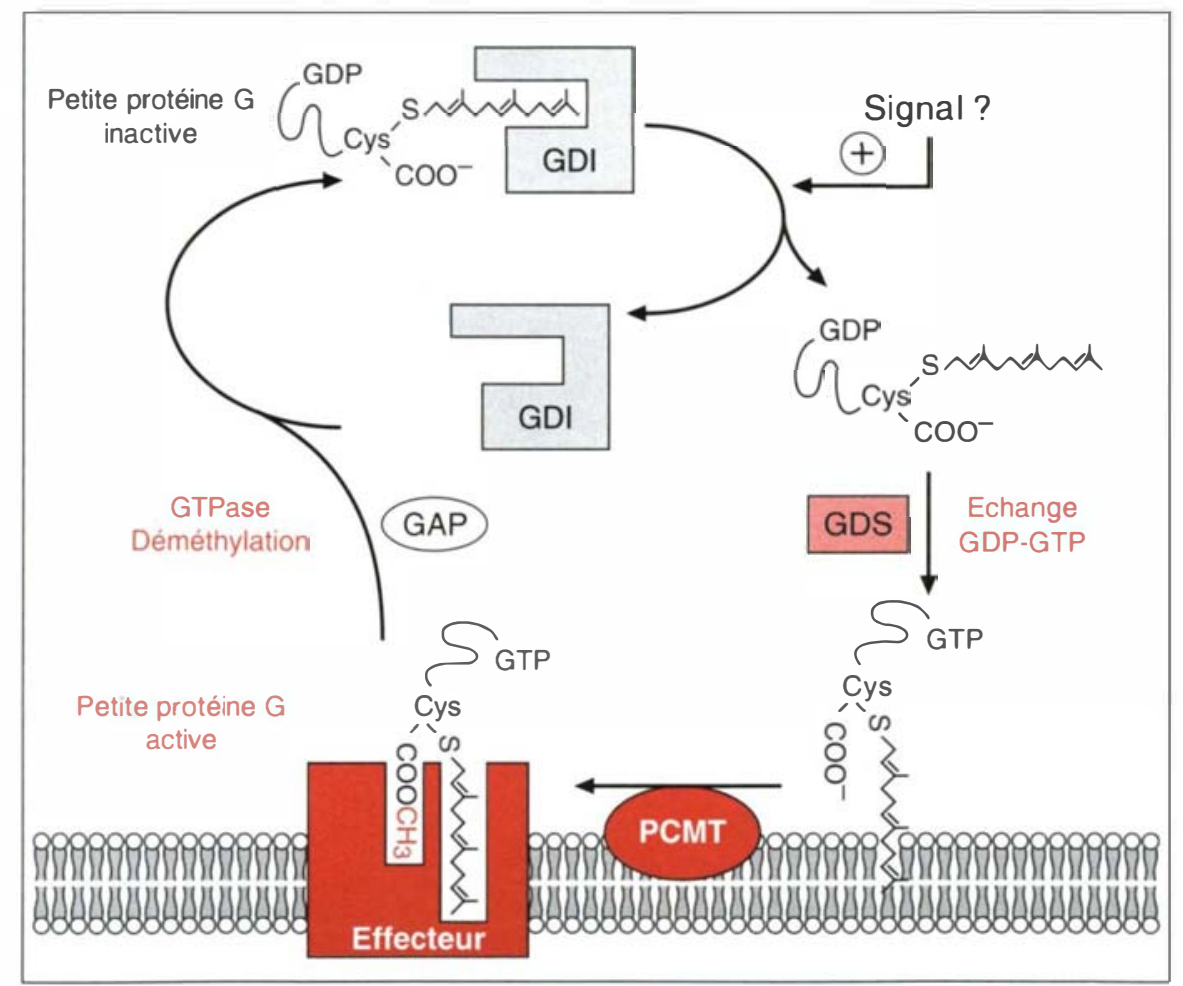

Figure 6. Régulation du processus d'activation des petites protéines $\mathbf{G}$. L'oscillation des petites protéines G entre l'état inactif (lié au GDP) et actif (lié au GTP) est contrôlée par les protéines régulatrices de la dissociation et de la liaison des nucléotides. Dans l'état inactif, un grand nombre de petites protéines $G$ sont complexées dans le cytosol des cellules avec des protéines inhibitrices de la dissociation du GDP (GDI). La stimulation de la cellule induit la dissociation de ces complexes, ce qui rend la protéine libre d'interagir avec une protéine stimulatrice de la dissociation du GDP (GDS). Le GDP est alors remplacé par le GTP, ce qui permet à la petite protéine $G$ d'atteindre l'état activé. Cette forme active de la protéine migre vers la membrane où elle est méthylée par une protéine carboxyle méthyltransférase (PCMT) et interagit avec une enzyme effectrice membranaire, provoquant une réponse de la cellule. Le signal est terminé par l'action d'une protéine GAP qui stimule l'activité GTPasique de la petite protéine $G$ et le retour à la forme liant le GDP. Cette forme est reconnue par la protéine GDI cytosolique qui séquestre la petite protéine $G$ de la membrane vers le cytosol dans un état inactif. La modification post-traductionnelle des petites protéines $G$ par isoprénylation et méthylation semble cruciale pour leur interaction avec les protéines GDI et GDS. 
protéines G cytosoliques qui, à la suite d'une stimulation, sont carboxyle méthylées et s'associent à la membrane où elles activent l'oxidase [48]. I'effet inhibiteur de l'AFC serait donc dû à une baisse de la liaison des petites protéines $G$ cytosoliques à la membrane [48]. L'ensemble de ces données suggère que la méthylation participe au recrutement des protéines cytosoliques isoprénylées du cytosol par la membrane où elles deviennent biologiquement actives. La méthylationdéméthylation pourrait donc servir de commutateur moléculaire pour contrôler le taux d'association des protéines isoprénylées à la membrane et ainsi l'activité des effecteurs de ces protéines [30, 44, 48].

\section{Conclusions}

Les rôles proposés pour les réactions de méthylation catalysées par les deux classes de carboxyle méthyltransférases des eucaryotes illustrent la diversité et l'importance de ces deux types d'enzymes. La réparation de résidus altérés présents dans la structure des protéines par les Lisoaspartate méthyltransférases a été observée au niveau de cellules très éloignées des mammifères dans l'évolution, suggérant que cette réaction est un mécanisme ancestral de défense de première ligne contre les dommages subis par les protéines au cours du vieillissement des cellules ou, encore, après exposition à des conditions de stress physiques ou métaboliques. Les données acquises récemment concernant les Cterminal methyltransferases suggèrent fortement la participation de la méthylation catalysée par cette classe d'enzyme à la régulation de l'activité des petites protéines $\mathbf{G}$. La caractérisation moléculaire de cette enzyme pourrait donc s'avérer extrêmement importante pour la compréhension des processus cellulaires auxquels participent ces protéines, tels que le transport vésiculaire, l'organisation du cytosquelette et la transduction des signaux hormonaux

TIRÉS A PART

\section{Summary}

Eukaryotic protein carboxyl methyltransferases: two distinct classes of enzymes

Protein carboxyl methyltransferases are ubiquitous enzymes that transfer a methyl group from $\mathrm{S}$ adenosyl-L-methionine (AdoMet) to some carboxyl groups of proteins. In eukaryotic cells, two distinct classes of these enzymes have been described to date. One of these, L-isoaspartate/D-aspartate methyltransferases, recognizes with high affinity damaged aspartic acid residues that originate mainly from spontaneous deamidation of asparagine residues and from racemisation of aspartate residues. These enzymes exist as molecular species of $27 \mathrm{kDa}$ and a number of isoforms have been identified. In all cases, these enzymes are non-specific and the methyl esters formed by their action are highly sensitive to moderate alkaline conditions. Numerous studies have suggested that these enzymes may be involved in the repair of the altered residues, as reflected by the repair of various isoaspartate-containing peptides and some proteins following incubation with AdoMet and purified carboxyl methyltransferases. The other class of methyltransferases, the C-terminal carboxyl methyltransferases, catalyze the methylation of the carboxyl group of isoprenylated cysteine residues located in the $\mathrm{C}$-terminal portion of various proteins, including members of the Ras superfamily of GTP-binding proteins. The post-translational modification of these proteins by isoprenylation and carboxyl methylation is supposed to play a major role in their association with cellular membranes, where they become physiologically active. In addition, recent studies have suggested that the methylation of isoprenylated proteins may play an important function in promoting specific interactions between these proteins and specific effectors located in the membrane.
Une conférence internationale INSERM-NIH “Nouvelles tendances thérapeutiques des hémoglobinopathies " se tiendra du 19 au 22 septembre 1994 à Paris, sous la présidence de Jean Rosa (INSERM U. 91)

- Comité d'organisation: V. Beuzard, F. Galactéros et P.-H. Roméo (INSERM U.91 - Fax: 498128 95), B. Lubin (USA - Fax: 1-510 428 3502), S. Piomelli (USA), L. Benjamin (USA).

L'objectif de la conférence est le développement des thérapeutiques des hémoglobinopathies et leur application clinique. En rassemblant les professionnels de différents domaines (cliniciens des hémoglobinopathies, spécialistes d'une thérapie, chercheurs), cette conférence facilitera la coopération et la mise en œuvre des thérapeutiques adaptées à chaque patient dans les pays développés ou en voie de développement.

- Programme et animateurs de sessions :

- Gènes de globine (F. Grosveld,

G. Fesenfeld, P.-H. Roméo)

- Modèles expérimentaux (O. Smithies, M. Trudel, T. Townes)

- Activation des gènes $\gamma$ de l'hémoglobine fœtale (G. Stamatoyannopoulos, A. Nienhuis)

- Thérapie génique (A. Bank, J.M. Heard, D. Valerio)

- Transplantation des cellules souches hématopoiétiques ( $E$. Gluckman, G. Lucarelli, H.E. Broxmeyer)

- Transfusion et infections (C. Poyart,

R. Girot, D. Winslow)

- Chélateurs de fer (C. Hershko, G. Hoffbrand, G. Brittenham)

- Progrès dans la prise en charge thérapeutique des patients ayant une hémoglobinopathie (S. Charache, A. Cao, E. Vichinsky, G. Serjeant) - Thérapies spécifiques (R. Hebbel, C. Brugnara, M. Narla, R. Nagel, A. Schechter)

- Éthique des thérapeutiques des hémoglobinopathies (F. Bunn, L. Luzzatto)

Les conférences ainsi que les communications sélectionées seront publiées pour la conférence, sous forme d'une coédition INSERM-John Libbey Eurotext dans la collection des Colloques INSERM sous le titre "Progrès thérapeutiques des hémoglobinopathies ".

Date limite d'appel à la communication orale et/ou affichée: 31 mars 1994

Renseignements et inscriptions (nombre de participants limité): Mme Didi Jobin, École Européenne d'Hématologie, Conférence INSERM-NIH, Centre Hayem, Hôpital Saint-Louis, 1, avenue Claude-Vellefaux, 75010 Paris

Télécopie : 42.41.14.70. 